PACS 61.30.Gd

\title{
Low voltage FLC for fast active matrix displays
}

\author{
Alexander Andreev, Tatiana Andreeva, Igor Kompanets* \\ P.N. Lebedev Physics Institute \\ 53, Leninsky prospect, 119991 Moscow, Russia \\ *E-mail:kompan@sci.lebedev.ru
}

\begin{abstract}
The process of FLC director reorientation in alternating electric field is considered for the case when interaction of FLC molecules with the substrates results in partial unwinding the helix structure and motion of domain walls. The hysteresis-free electrooptical response as fast as $50 \ldots 70 \mu \mathrm{s}$ was achieved in the FLC cell of $1.3-\mu \mathrm{m}$ thickness in the electric field $1 \mathrm{~V} / \mu \mathrm{m}$. This result is very important for the practical use of FLC in fast active matrix displays.
\end{abstract}

Keywords: FLC, fast active matrix display, electrooptical response, reorientation of direct.

Manuscript received 18.12.09; accepted for publication 08.07.10; published online 30.09.10.

\section{Introduction}

In FLC cell the helix pitch $p_{0}$ and elastic module $K_{\varphi}$ determining the deformation of the azimuth angle $\varphi$ of the director orientation are related with the dispersion coefficient of anchoring energy $W_{Q}$ and cell thickness $d$ by the expression $K_{\varphi} \cdot q_{0}^{2} \approx W_{Q} / d$, where $q_{0}=2 \pi / p_{0}$ [1]. The interaction between molecules and surface results in partial unwinding of the helix structure.

Further the process of FLC director reorientation and domain wall motion in alternating electric field is considered for the case of portion unwinding of FLC helix structure. The director of used FLC is oriented planar, and the dispersion coefficient of anchoring energy is $W_{Q}=0.05 \mathrm{Erg} / \mathrm{cm}^{2}$. In such a case, the partial unwinding of a FLC helix structure with the helix pitch $0.45 \mu \mathrm{m}$ is observed in the FLC cell of 1.3 to $1.5-\mu \mathrm{m}$ thickness.

The electrooptical response time in dependence on the electric field, frequency, boundary conditions, spontaneous polarization and FLC viscosity is studied.

\section{Motion of domain walls}

The domains, where dipole moments of molecules are not oriented along the field are unstable. Thereby in regions, in which the dipole moments of molecules are placed in energy optimal manner, some regions appear where the azimuth angle $\varphi$ has a maximum divergence from $\varphi=0$ (or $\varphi=\pi)$ originally. This process results in appearance of transient domains, where these dipole moments are directed against the field separate the regions with dipole orientation along the field. These domains are coupled states of two $180^{\circ}$ domain walls of different signs [2].

Domain walls begin to move when the critical value of the electric field is reached. The domain walls of different sign move to opposite directions in such a way that the volume of domains with the optimal energy increases at the expense of the non-optimal energy domains. As a result, the azimuth angle $\varphi$ in all smectic layers becomes the same and equal 0 or $\pi$ (depending on the field direction), and the vector $\boldsymbol{P}_{\boldsymbol{S}}$ is oriented along the field. Inversion of the electric field sign induces the creation of transient domains, and the process begins again.

The equation of wall motion along $Z$ coordinate can be written as follows $[2,3]$ :

$\gamma_{\varphi} \frac{\partial \varphi}{\partial t}=K_{\varphi} \frac{\partial^{2} \varphi}{\partial z^{2}} \pm\left|P_{S} \| E\right| \sin \varphi+\frac{2 W_{Q}}{d} \sin 2 \varphi+\frac{W_{P}}{d} \sin \varphi$.

The solution of this equation describes the soliton motion along $Z$ coordinate with the velocity 
$v=\sqrt{\frac{K_{\varphi} d}{2 W_{Q}}} \cdot \frac{P_{S} E d+W_{P}}{\gamma_{\varphi} d}$.

\section{Electrooptical response time and dissipation coefficient role}

FLC properties can be characterized by the rotational viscosity $\gamma_{\varphi}$ and modulus of transverse elasticity $\mu$. A character of the FLC director reorientation under the external electric field $E$ depends on which of energy dissipation coefficients - rotational or shift (shear) viscosity predominates [2].

When the dissipation coefficient is rotational viscosity $\gamma_{\varphi}$, director reorientation corresponding to $180^{\circ}$ change of the azimuth angle $\varphi$ takes place simultaneously in all FLC smectic layers (volume switching mode) with the character time $\tau_{R} \sim \gamma_{\varphi} /\left(\boldsymbol{P}_{S} \boldsymbol{E}\right)$, and this time does not depend on the electric field frequency [3]. Fig. 1 (curves 1 and 2) shows that the electrooptical response time $\tau_{0.1-0.9}$ does not depend on the electric field frequency $f$ within the range $f \leq 300 \mathrm{~Hz}$. The optical transmission of this cell and contrast ratio $C$ (curve 3 ) do not depend on frequency in this range, too.

\section{Electrooptical response time and control voltage frequencies}

For the electric field of low frequency $(f<300 \mathrm{~Hz})$ and strength $E<4 \mathrm{~V} / \mu \mathrm{m}$, director reorientation in FLC volume is defined by the rotation viscosity coefficient (Fig. 2, curve 1). In this case, the reverse electrooptical response $1 / \tau_{0.1-0.9}$ increases proportionally to the electric field intensity.

When the electric field frequency is higher than $300 \mathrm{~Hz}$, the director in FLC volume reorients because of domain wall motion, and then the reverse time $1 / \tau_{0.1-0.9}$ changes proportionally to the velocity of wall motion (Fig. 2, curves 2 and 3). The reverse time depending on the wall motion velocity linearly increases.

The dependence of light transmittance $I$ on control voltage for low and high frequencies at voltage increase and decrease is the same and practically hysteresis-free (Fig. 3)

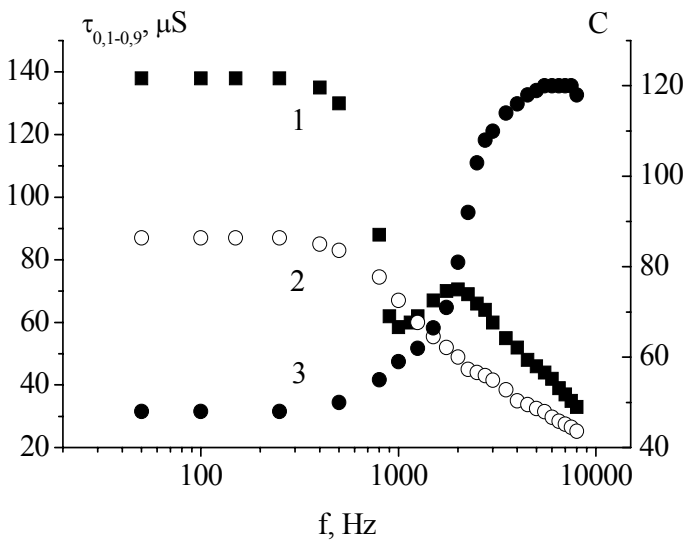

Fig. 1. Frequency dependences of the electrooptical response time $\tau_{0.1-0.9}$ at $E=2 \mathrm{~V} / \mu \mathrm{m}$ (curve 1) and $3 \mathrm{~V} / \mu \mathrm{m}$ (curve 2), and the contrast ratio (curve 3) at $E=3 \mathrm{~V} / \mu \mathrm{m}$. FLC cell has no dielectric coatings, cell thickness is $1.3 \mu \mathrm{m}$ and FLC helix pitch is $0.45 \mu \mathrm{m}$.
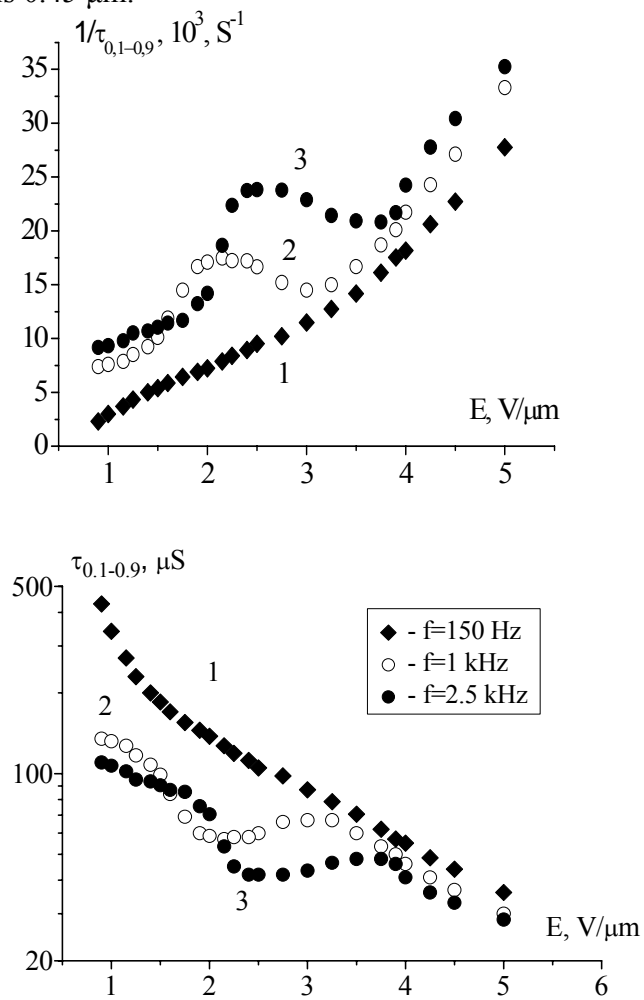

Fig. 2. Electric field dependences of the electrooptical response at control voltage frequencies: $1-150 \mathrm{~Hz} ; 2-1 \mathrm{kHz}, 3-$ $2.5 \mathrm{kHz}$. No dielectric coatings, FLC thickness is $1.3 \mu \mathrm{m}$ and helix pitch is $0.45 \mu \mathrm{m}$.
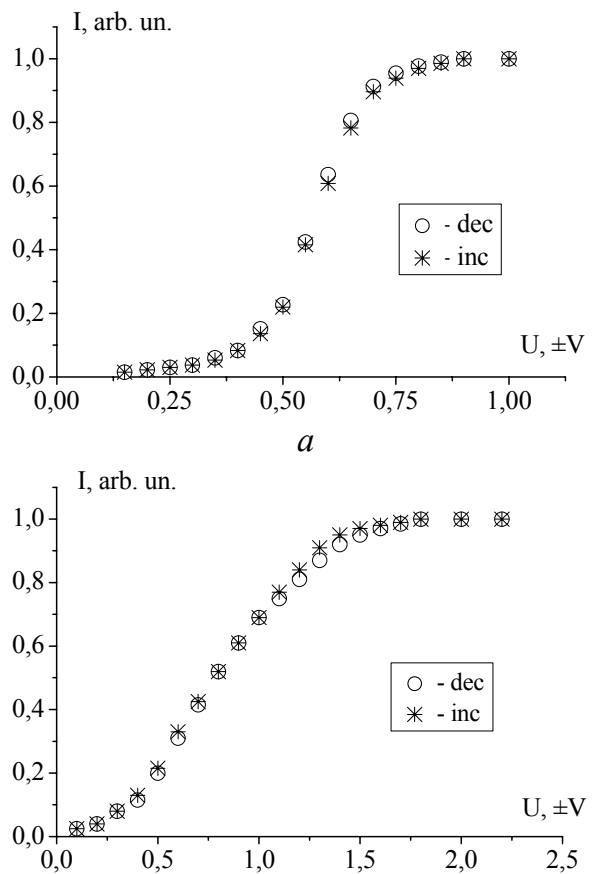

(C) 2010, V. Lashkaryov Institute of Semiconductor Physics, National Academy of Sciences of Ukraine 


\section{$b$}

Fig. 3. The light transmittance versus the control voltage (meander) for the pulse frequency $200 \mathrm{~Hz}$ (a) and $1 \mathrm{kHz}(\mathrm{b})$. The FLC layer thickness is $1.3 \mu \mathrm{m}$. One of substrates is coated with dielectric.

\section{Electrooptical response time and dielecric coating}

When one of substrates is coated with a dielectric layer (polar boundary conditions), FLC structure is polarized under influence of anchoring energy polar part $\left(W_{P} \neq 0\right)$ that results in helix deformation (partial unwinding) and appearance of structural domains without the electric field. Coefficient $W_{P}$ increases almost 3 times, which leads to restructuring the domain walls. As a result, the electrooptical mode appears at the frequency $f=200 \mathrm{~Hz}$ of the control voltage (Fig. 4, curve 2), and this mode corresponds to the director reorientation because of domain walls motion.

The minimal electrooptical response time of 50 to $70 \mu \mathrm{s}$ was achieved in FLC cell of $1.3-\mu \mathrm{m}$ thickness at the field strength close to $1 \mathrm{~V} / \mu \mathrm{m}$.

\section{Electrooptical response time and spontaneous polarization}

The velocity of domain wall motion is proportional to the ratio $P_{S} / \gamma_{\varphi}$ and the polar part of anchoring energy $W_{P}$. Increasing the spontaneous polarization $P_{S}$ and decreasing the rotation viscosity $\gamma_{\varphi}$ under polar boundary conditions result in increase of the domain wall motion velocity, and hence the reverse response time $1 / \tau_{0.1-0.9}$ (Fig. 5).

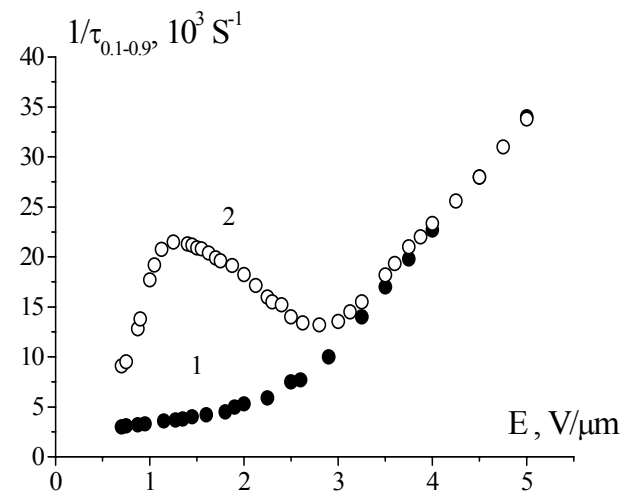

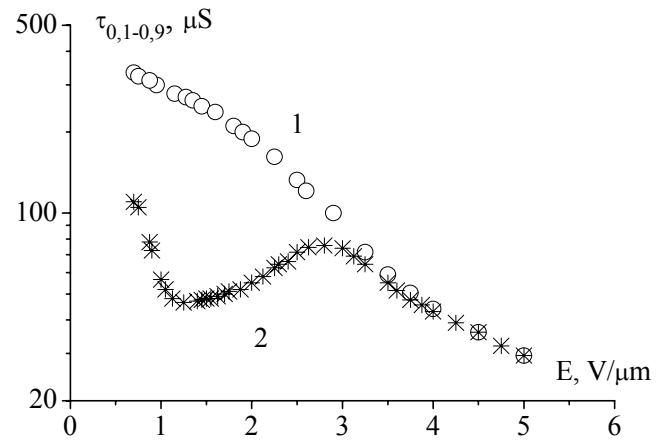

Fig. 4. Electric field dependences of the reverse electrooptical response time for FLC cells without a dielectric coating (curve 1) and with dielectric coating on one of substrates (curve 2); $d=1.3 \mu \mathrm{m}, f=200 \mathrm{~Hz}, p_{0}=0.45 \mu \mathrm{m}$.

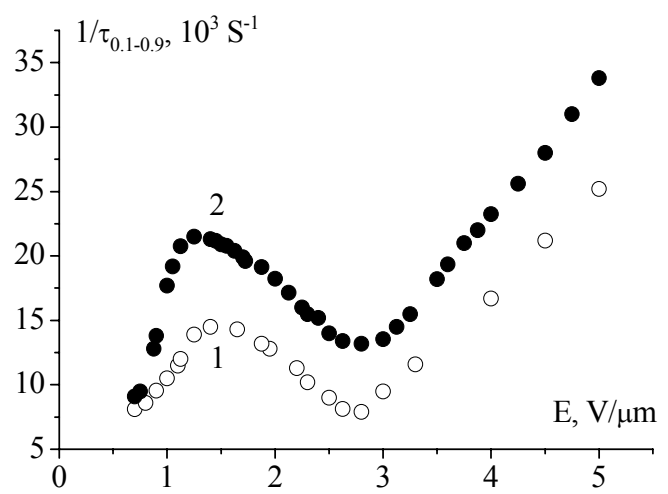

Fig. 5. Field dependences of the reverse electrooptical response time for FLC cells with dielectric coating on one of substrates for: $1-P_{S}=100 \mathrm{nC} / \mathrm{cm}^{2}$ and $\gamma_{\varphi}=1.3$ Poise; $2-P_{S}=$ $80 \mathrm{nC} / \mathrm{cm}^{2}$ and $\gamma_{\varphi}=0.55$ Poise. $d=1.4 \mu \mathrm{m}, f=200 \mathrm{~Hz}, p_{0}=$ $0.45 \mu \mathrm{m}$

Location of the maximum and character of the dependence $1 / \tau_{0.1-0.9}(E)$ is not changed practically, when the ratio $P_{S} / \gamma_{\varphi}$ increases. The increase in the ratio $P_{S} / \gamma_{\varphi}$ almost 2 times results in the decrease of the electrooptical response time $\tau_{0.1-0.9}$ more than 1.5 times (Fig. 5, curve 2).

\section{Conclusion}

The process of FLC director reorientation under the alternating electric field is considered for the case when portion unwinding of the helix structure arises from the interaction of FLC molecules with substrates, and domain wall motion results in this director reorientation.

It has been shown that the character of FLC director reorientation under the electric field depends on which of coefficients of energy dissipation - rotational or shift viscosity predominates. When the shift viscosity begins to define the electrooptical response time during director reorientation, a strong frequency dependence of $\tau_{0.1-0.9}$ takes place.

It has been shown experimentally that increasing the electric field frequency or polarity of boundaries 
(when the frequency is constant) gives rise to increasing the domain wall motion velocity. As a result, the electrooptical response time $\tau_{0.1-0.9}$ decreases at least by the factor 3 in low electric fields ( 1 to $2 \mathrm{~V} / \mu \mathrm{m})$.

For low frequency electric field and polar boundary conditions, the electrooptical response time that is proportional to domain walls velocity depends on the ratio of spontaneous polarization and rotational viscosity. Increasing the ratio $P_{S} / \gamma_{\varphi}$ results in the decreasing the electrooptical response time $\tau_{0.1-0.9}$.

The dependence of light transmittance on the control voltage for varios frequencies at voltage increase and decrease is the same and practically hysteresis-free.

These results are very important for the practical use of ferroelectric LC in fast active matrix displays. Really, in FLC layer of $1.3 \mu \mathrm{m}$ thickness the response time of $50 \ldots 70 \mu \mathrm{s}$ under the electric field close to $1 \mathrm{~V} / \mu \mathrm{m}$ was reached. This time is almost two orders less than in displays based on nematic LC (a few milliseconds under a few volts).

\section{Acknowledgements}

Authors thank the Russian Foundation for Basic Research for the support of grants 08-07-13554, 07-0200785 and 07-07-91582 as well as thank the Russian Academy of Sciences for its support of this work on the Program "Polyfunctional materials for molecular electronics".

\section{References}

1. V.G. Chigrinov, Liquid Crystal Devices: Physics and Applications. Artech House, London, 1999.

2. A. Andreev, E. Pozhidaev, T. Fedosenkova, I. Kompanets, Yu. Shumkina, Dynamics of the domain wall motion in FLC display cell // Proc. SPIE 6637, p. 74-78 (2007).

3. M.A. Handschy, N.A. Clark, S.T. Lagerwall // Phys. Rev. Lett. 51, p. 471 (1983). 